To be presented at: 15th Biennial ASME Vibrations Conference Boston, Massachusetts

September 17-21, 1995

N95- 32692

$$
\operatorname{cin} x 4
$$

\title{
DYNAMIC MODELLING AND RESPONSE CHARACTERISTICS OF A MAGNETIC BEARING ROTOR SYSTEM WITH AUXILIARY BEARINGS
}

\author{
April M. Free and George T. Flowers \\ Department of Mechanical Engineering \\ Auburn University \\ Auburn, AL 36849 \\ Victor S. Trent \\ Department of Electrical Engineering \\ Auburn University \\ Auburn, AL 36849
}

\begin{abstract}
Auxiliary bearings are a critical feature of any magnetic bearing system. They protect the soft iron core of the magnetic bearing during an overload or failure. An auxiliary bearing typically consists of a rolling element bearing or bushing with a clearance gap between the rotor and the inner race of the support. The dynamics of such systems can be quite complex. It is desired to develop a rotordynamic model which describes the dynamic behavior of a flexible rotor system with magnetic bearings including auxiliary bearings. The model is based upon an experimental test facility. Some simulation studies are presented to illustrate the behavior of the model. In particular, the effects of introducing sideloading from the magnetic bearing when one coil fails is studied. These results are presented and discussed.
\end{abstract}

\section{NOMENCLATURE}

$C=$ damping, $\mathrm{N}-\mathrm{s} / \mathrm{m}$

$C_{L}=$ clearance of auxiliary bearing, $\mathrm{m}$

$D=$ nominal gap thickness, $\mathrm{m}$

$\mathbf{F}_{X}=$ external force vector acting on the rotor in

$\mathrm{X}$ direction, $\mathrm{N}$

$\mathrm{F}_{Y}=$ external force vector acting on the rotor in

$\mathrm{Y}$ direction, $\mathrm{N}$

$\mathrm{G}=$ gravitational acceleration, $\mathrm{m} / \mathrm{s}^{2}$
$I=$ rotor inertia matrix

$i=$ current, amp

$K=$ stiffness, $\mathrm{N} / \mathrm{m}$

$k=$ gain value

$\mathrm{L}=$ equivalent circuit length

$M=$ mass, $\mathrm{kg}$

$m=$ total number of nodes

$N=$ total number of modes considered

$N_{B 1}=$ node number at rotor left end

$N_{B 2}=$ node number at $\mathrm{AMB}$

$N_{B 3}=$ node number at auxiliary bearing

$N_{i m b}=$ node number at imbalance location

$N_{T}=$ number of turns of wire per coil

$\mathbf{Q}_{X}=$ rotor modal coordinate vector in $\mathrm{X}$ direction

$Q_{Y}=$ rotor modal coordinate vector in $Y$ direction

$R_{R}=$ radius of rotor journal, $\mathrm{m}$.

$R_{S}=$ radius of auxiliary bearing bore, $\mathrm{m}$.

$t=$ time, $s$

$t_{f}=$ fail time, $s$

$v=$ voltage, volt

$v_{r}=$ relative velocity at auxiliary bearing rotor/stator contact point

$\mathrm{X}_{R}=$ rotor physical coordinate vector in $\mathrm{X}$ direction

$\mathrm{X}_{S}=$ stator physical coordinate vector in $\mathrm{X}$ direction

$Y_{R}=$ rotor physical coordinate vector in $\mathrm{Y}$ direction

$Y_{S}=$ stator physical coordinate vector in $Y$ direction

$\alpha=$ acceleration, $\mathrm{rad} / \mathrm{sec}^{2}$

$\delta=$ dynamic clearance 
$\Gamma=\Psi^{T} \mathbf{I} \Psi$

$\Phi=$ rotor free-free modal displacement matrix

$\Psi=$ rotor free-free modal rotation matrix

$\psi=$ imbalance vector

$\mu_{o}=$ permeability of free space

$\mu_{r}=$ permeability of silicon steel

$\Omega=$ rotor operating speed, $\mathrm{rad} / \mathrm{s}$

$\tau=$ time constant

$\theta=$ angular position of the shaft

$\zeta=$ modal damping coefficient

\section{Subscripts}

$a=$ first

$B=$ auxiliary bearing

$b=$ second

$C=$ contact

$g=$ gain constant

$h=$ horizontal

$l=$ left bearing

$r=$ rate constant

$v=$ vertical

$x=\mathrm{x}-$ direction

$y=y-$ direction

$b i=$ bias

$1=$ top magnet

$2=$ right magnet

3 = bottom magnet

$4=$ left magnet

\section{Superscripts}

$a=$ current amplifier

$c=$ controller

$l=$ lead

$p=$ sensor amplifier

\section{INTRODUCTION}

In recent years, the use of active magnetic bearings (AMB) for turbomachinery support has been an area of interest in both academia and in industry. Magnetic bearings provide the potential for significant improvements over other types of rotor supports, including elimination of wear, bearing friction-related energy losses and a means of actively suppressing rotor vibration. A critical feature of any magnetic bearing supported rotor system is auxiliary bearings to protect the soft iron core from rotor contact (and subsequent damage) during an overload or failure of the AMB. The present work is concerned with developing a suitable model and investigating the dynamic behavior of such a system.

\section{EXPERIMENTAL TEST FACILITY}

The model and control system development presented in this study is for an experimental test facility. The apparatus consists of a radial magnetic bearing that is supporting the right end of a rotor. The left end of the rotor is supported by a ball bearing suspended in a frame by four springs. A photograph and the corresponding schematic diagram are shown in Figure 1.

The apparatus has two basic components: the rotor shaft and the magnetic bearing. The shaft is made of steel and is 0.0098 meters in diameter and 0.4572 meters in length. A steel disk of diameter $7.62 \mathrm{~cm}$, thickness $2.54 \mathrm{~cm}$, and mass $0.810 \mathrm{~kg}$ is placed at the midpoint of the bearing span. Threaded holes on the disk allow for imbalance to be added to the system. The rotor is driven by a variable speed motor with a controller. Shaft vibration is measured using eddy current proximity displacement sensors fixed to measure displacement in both the vertical and horizontal directions.

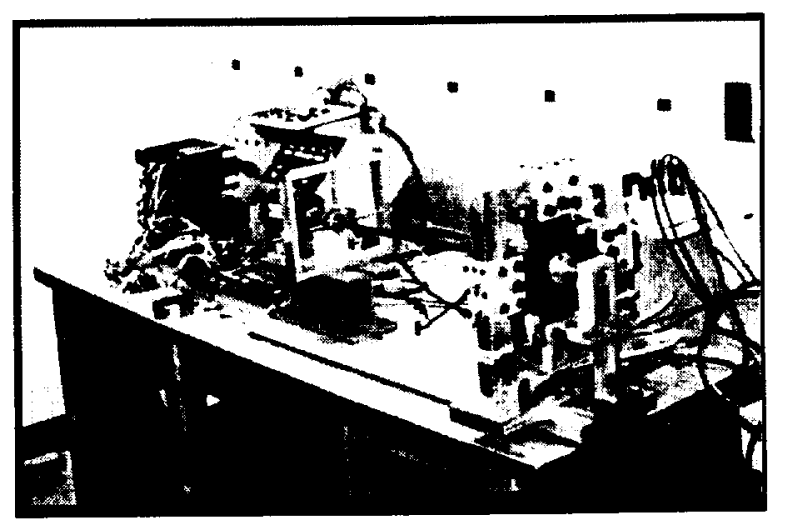

Figure 1.a Experimental Test Facility

The magnetic bearing is based upon a design described in Humphris, et al (1988). The basic parameters for the bearing are shown in Table 1. The bearing consists of four electromagnets equally spaced around a soft-iron core. A photograph and schematic diagram of the bearing are shown in Figure 2.

\section{SIMULATION MODEL}

A simulation model was developed for the rotor system described above. The model has three principal components - the rotor, magnetic bearing, and electronics. The governing equations for ach are shown below. 


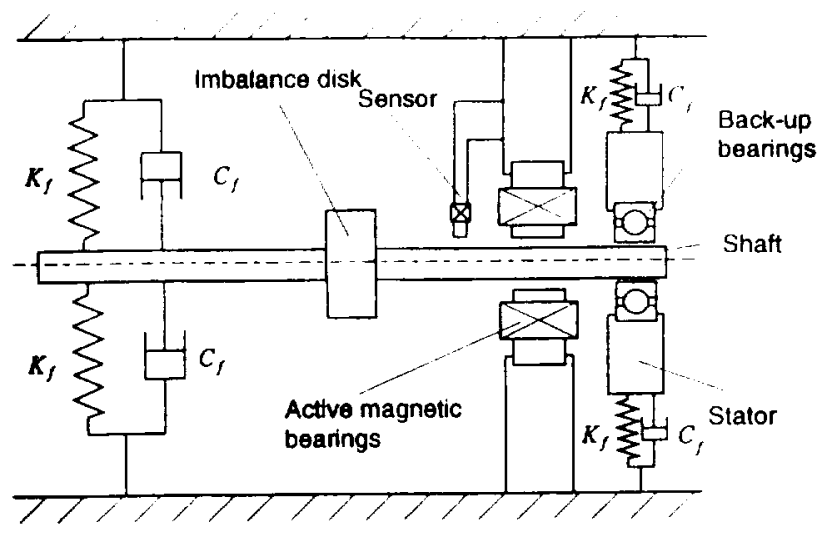

Figure 1.b Schematic Diagram of Experimental Test Facility

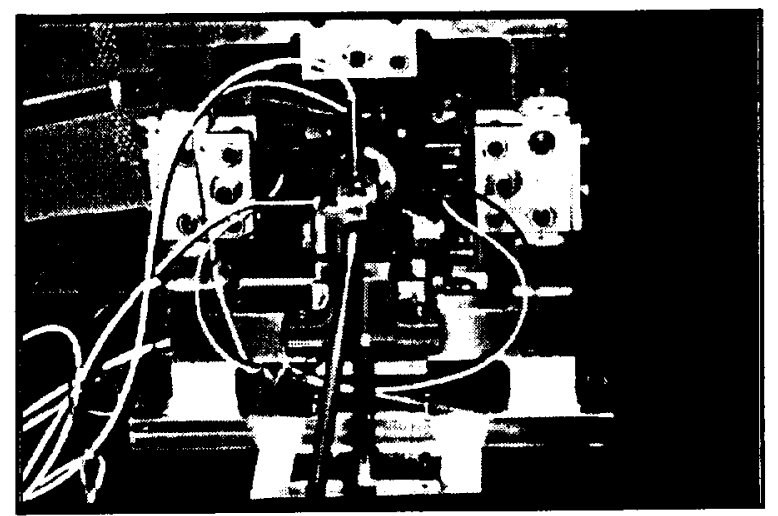

Figure 2.a Magnetic Bearing Assembly

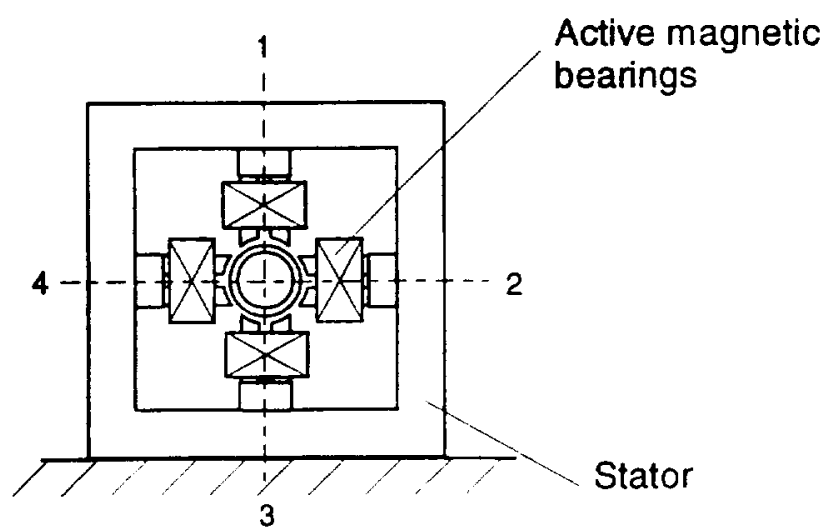

Figure 2.b Schematic Diagram of Magnetic Bearing
The rotor is modelled using the free-free bending mode shapes and natural frequencies obtained through finite element analysis. The finite element code uses 11 stations and the first four modes (two rigid body and two flexible modes) are included in the simulation model. The rotor equations of motion can be expressed in terms of modal coordinates as

$$
\begin{aligned}
& \ddot{\mathbf{Q}}_{x}+\Omega \Gamma \dot{\mathrm{Q}}_{y}+\omega_{n}^{2} \mathbf{Q}_{x}+\Phi^{T} \mathbf{F}_{x}=0 \\
& \ddot{\mathbf{Q}}_{y}-\Omega \Gamma \dot{\mathbf{Q}}_{x}+\omega_{n}^{2} \mathbf{Q}_{y}+\Phi^{T} \mathbf{F}_{y}=0
\end{aligned}
$$

The auxiliary bearing consists of a ball bearing with a clearance between the rotor and the stator. The governing equations are

$$
\begin{aligned}
& M_{B} \ddot{X}_{B}+C_{B x} \dot{X}_{B}+K_{B x} X_{B}=F_{x, a u x},(1 . c{ } \\
& M_{B} \ddot{Y}_{B}+C_{B y} \dot{Y}_{B}+K_{B y} Y_{B}=F_{y, a u x}
\end{aligned}
$$

where

$$
\begin{aligned}
\mathbf{F}_{x, l i n}\left(N_{b 1}\right) & =\Phi_{N_{b l}}\left(K_{l x} \mathbf{Q}_{x}+C_{l x} \dot{\mathbf{Q}}_{x}\right) \\
\mathbf{F}_{y, l i n}\left(N_{b 1}\right) & =\Phi_{N_{b 1}}\left(K_{l y} \mathbf{Q}_{y}+C_{l y} \dot{\mathbf{Q}}_{y}\right) \\
\mathbf{F}_{x, \text { imb }}\left(N_{i m b}\right) & =\Omega^{2} \psi_{6} \cos \theta+\alpha \psi_{6} \sin \theta, \\
\mathbf{F}_{y, i m b}\left(N_{i m b}\right) & =\Omega^{2} \psi_{6} \sin \theta-\alpha \psi_{6} \cos \theta, \\
\mathbf{F}_{x, a u x}\left(N_{b 3}\right) & =\Phi_{N_{b 3}}\left(\mathbf{F}_{N} \cos \beta-\mathbf{F}_{R} \sin \beta\right) \\
\mathbf{F}_{y, a u x}\left(N_{b 3}\right) & =\Phi_{N_{b 3}}\left(\mathbf{F}_{N} \sin \beta+\mathbf{F}_{R} \cos \beta\right) \\
\mathbf{F}_{\text {grav }} & =\boldsymbol{\Phi}^{T} M G \\
\mathbf{F}_{N} & =\phi K_{c}(\delta-\mathbf{D}) .
\end{aligned}
$$

If $v_{r}=0$, then $\mathbf{F}_{R}=$ required static friction force to satisfy this condition. If $v_{r} \neq 0$ or if required static friction force $>\mu_{s} F_{N}$, then $\mathbf{F}_{R}=\mu_{k} \mathbf{F}_{N}$.

$$
\begin{aligned}
& \mathbf{F}_{\mathbf{x}}=\mathbf{F}_{x, \text { iin }}+\mathbf{F}_{x, i m b}+\mathbf{F}_{x, a u x}-\mathbf{F}_{x, a m b} \\
& \mathbf{F}_{\mathbf{y}}=\mathbf{F}_{y, \text { lin }}+\mathbf{F}_{y, i m b}+\mathbf{F}_{y, a u x}-\mathbf{F}_{y, a m b}+\mathbf{F}_{g r a v}
\end{aligned}
$$

where 


$$
\begin{aligned}
\sin (\beta)= & \frac{\mathbf{Y}_{R}-\mathbf{Y}_{S}}{\sqrt{\left(\mathbf{X}_{R}-\mathbf{X}_{S}\right)^{2}+\left(\mathbf{Y}_{R}-\mathbf{Y}_{S}\right)^{2}},} \\
\cos (\beta)= & \frac{\mathbf{X}_{R}-\mathbf{X}_{S}}{\sqrt{\left(\mathbf{X}_{R}-\mathbf{X}_{S}\right)^{2}+\left(\mathbf{Y}_{R}-\mathbf{Y}_{S}\right)^{2}},} \\
\delta= & \sqrt{\left(\Phi \mathbf{Q}_{x}-X_{b}\right)^{2}+\left(\Phi Q_{y}-Y_{b}\right)^{2}}, \\
\phi= & 1 \text { if } \delta>\Delta \\
& 0 \text { otherwise } \\
\mathbf{Q}_{x}= & \Phi^{T} \mathbf{X}_{r}, \\
\mathbf{Q}_{y}= & \Phi^{T} \mathbf{Y}_{r},
\end{aligned}
$$

implemented using analog circuitry. The controller is modelled as

$$
\begin{aligned}
& \frac{d v_{v}^{c}}{d t}=k_{g} v_{v}^{p} \tau^{c}+\left(k_{g}+k_{r}\right) \frac{v_{v}^{p}}{d t}-\tau^{c} v_{v}^{c} \\
& \frac{d v_{h}^{c}}{d t}=k_{g} v_{h}^{p} \tau^{c}+\left(k_{g}+k_{r}\right) \frac{v_{h}^{p}}{d t}-\tau^{c} v_{h}^{c}
\end{aligned}
$$

The control circuitry also includes a lead network. The governing equations are

$$
\frac{d v_{v}^{l}}{d t}=\frac{1}{\tau_{a}^{l}} v_{v}^{c}+\frac{d v_{v}^{c}}{d t}-\frac{1}{\tau_{b}^{l}} v_{v}^{l}
$$

with

$$
\begin{aligned}
& \mathbf{X}_{r}=\left\{X_{r 1}, X_{r 2}, \ldots, X_{r m}\right\}^{T} \\
& Y_{r}=\left\{Y_{r 1}, Y_{r 2}, \ldots, Y_{r m}\right\}^{T}
\end{aligned}
$$

The physical displacements of the rotor at the bearing and imbalance locations can be obtained using the following coordinate transformation:

$$
\begin{aligned}
X_{r k}= & \sum_{i=1}^{N} \Phi_{k i} Q_{x i}, \\
Y_{r k}= & \sum_{i=1}^{N} \Phi_{k i} Q_{y i}, \\
& \left(k=N_{b 1}, N_{b 2}, N_{b 3}, N_{i m b}\right)
\end{aligned}
$$

$$
\frac{d v_{h}^{l}}{d t}=\frac{1}{\tau_{a}^{l}} v_{h}^{c}+\frac{d v_{h}^{c}}{d t}-\frac{1}{\tau_{b}^{l}} v_{h}^{l}
$$

Four current amplifiers (one for each coil) supply current to the magnetic bearing. They are modelled as

$$
\begin{aligned}
& \frac{d i_{1}^{a}}{d t}=-\frac{1}{\tau^{a}} i_{1}^{a}+\frac{1}{\tau^{a}} k_{1}^{a} v_{v}^{l} \\
& \frac{d i_{2}^{a}}{d t}=-\frac{1}{\tau^{a}} i_{2}^{a}+\frac{1}{\tau^{a}} k_{2}^{a} v_{v}^{l}
\end{aligned}
$$

$$
\frac{d i_{3}^{a}}{d t}=-\frac{1}{\tau^{a}} i_{3}^{a}+\frac{1}{\tau^{a}} k_{3}^{a} v_{h}^{l}
$$

$$
\begin{aligned}
& \frac{d v_{v}^{p}}{d t}=\frac{k^{p} y-v_{v}^{p}}{\tau^{p}} \\
& \frac{d v_{h}^{p}}{d t}=\frac{k^{p} x-v_{h}^{p}}{\tau^{p}}
\end{aligned}
$$$$
\frac{d i_{4}^{a}}{d t}=-\frac{1}{\tau^{a}} i_{4}^{a}+\frac{1}{\tau^{a}} k_{4}^{a} v_{h}^{b}
$$

The forces supplied by the magnetic bearing to the rotor are assumed to be decoupled in the horizontal and vertical directions. The effects of flux saturation are included in the model based upon the approach described by Lewis (1993). The equations for these forces are

The active control system is based upon a proportional-derivative (PD) control law and 


$$
\begin{array}{r}
\mathrm{F}_{x, \mathrm{a} m b}=\mu_{o} A N_{T}^{2}\left(\frac{i_{2}^{2}}{\left(2\left(D-x_{N_{B 2}}\right)+\frac{L}{\mu_{r}}\right)^{2}}\right. \\
\left.-\frac{i_{4}^{2}}{\left(2\left(D+x_{N_{B 2}}\right)+\frac{L}{\mu_{r}}\right)^{2}}\right)
\end{array}
$$

$$
\begin{array}{r}
\mathbf{F}_{y, a m b}=\mu_{o} A N_{T}^{2}\left(\frac{i_{1}^{2}}{\left(2\left(D-y_{N_{B 2}}\right)+\frac{L}{\mu_{r}}\right)^{2}}\right. \\
\left.-\frac{i_{3}^{2}}{\left(2\left(D+y_{N_{B 2}}\right)+\frac{L}{\mu_{r}}\right)^{2}}\right)
\end{array}
$$

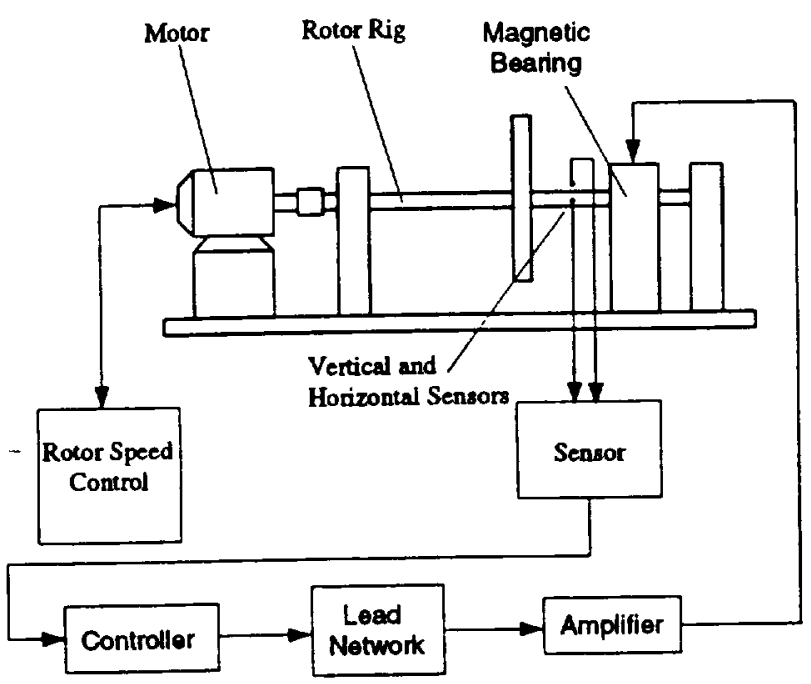

Figure 3 Block Diagram of Simulation Model

\section{DISCUSSION}

Using the rotordynamic model described in the preceding paragraphs, a study of the combined dynamics of a magnetic bearing supported rotor with auxiliary bearings was per-

\begin{tabular}{|c|c|c|}
\hline Parameter & Value & Units \\
\hline $\mathrm{K}_{\mathrm{lx}}, \mathrm{K}_{\mathrm{ly}}$ & 17,510 & $\mathrm{~N} / \mathrm{m}$ \\
\hline $\mathrm{C}_{l_{x}}, \mathrm{C}_{y_{y}}$ & 2.0 & $\mathrm{~N}-\mathrm{s} / \mathrm{m}$ \\
\hline$\overline{K_{C}}$ & 87,557 & $\mathrm{~N} / \mathrm{m}$ \\
\hline $\mathrm{C}_{\mathrm{C}}$ & 0 & $\mathrm{~N}-\mathrm{s} / \mathrm{m}$ \\
\hline$\overline{\mathrm{K}_{\mathrm{B}}}$ & 17,510 & $\mathrm{~N} / \mathrm{m}$ \\
\hline $\mathrm{C}_{\mathrm{Bx}}, \mathrm{C}_{\mathrm{By}}$ & 2.0 & $\mathrm{~N}-\mathrm{s} / \mathrm{m}$ \\
\hline $\mathrm{M}_{\mathrm{B}}$ & 0.033 & $\mathrm{~kg}$ \\
\hline$\psi_{6}$ & $3.0 \times 10^{-6}$ & $\mathrm{~kg}-\mathrm{m}$ \\
\hline$k^{p}$ & $7.8 \times 10^{3}$ & - \\
\hline$k_{g}$ & 13 & $\overline{-}$ \\
\hline$k_{r}$ & 23 & - \\
\hline$k^{a}$ & 0.45 & $\operatorname{amp} / v$ \\
\hline$\overline{\tau^{p}}$ & $1.59 \times 10^{-5}$ & - \\
\hline$\tau_{a}^{l}$ & $1.8 \times 10^{-3}$ & - \\
\hline$\tau_{b}^{l}$ & $1.59 \times 10^{-4}$ & - \\
\hline$\tau^{a}$ & $5.31 \times 10^{-5}$ & - \\
\hline$\overline{\mathrm{A}}$ & $3.42 \times 10^{-4}$ & $\mathrm{~m}^{2}$ \\
\hline $\mathbf{N}_{\mathrm{T}}$ & 164 & - \\
\hline$\overline{\mathrm{D}}$ & $0.9 \times 10^{-3}$ & $\mathbf{m}$ \\
\hline$\mu_{0}$ & $1.26 \times 10^{-6}$ & - \\
\hline$v_{1}^{b}$ & 4.2 & volt \\
\hline$v_{2}^{b i}$ & 1.9 & volt \\
\hline$v_{3}^{b i}$ & 1.0 & volt \\
\hline$v_{4}^{b i}$ & 1.9 & volt \\
\hline
\end{tabular}
formed. The parameters used in this work were identified from experimental evaluation of the test rig and are shown in Table 1. All of the
Table 1 Simulation Model Parameters

responses illustrated in the figures are for this base parametric configuration, unless otherwise indicated. Figure 3 shows the variation of system natural frequencies with rotor speed for the base parametric configuration (without auxiliary bearing contact). The rotor speed of 200 $\mathrm{rad} / \mathrm{sec}$ was selected as a reasonable value near a critical speed in order to illustrate a worst case behavior of the system.

Figure 4 shows the rotor response under normal operating conditions. The horizontal and vertical responses are of the same amplitude and frequency at steady state. The response consists of a transient region of about 0.5 seconds in length followed by steady synchronous oscillations. The initial conditions are set so that the rotor contacts the auxiliary bearing during the transient phase of the oscillations. The rotor vibrates downward, contacts the auxiliary bearing, bounces several times, and then settles into a steady synchronous whirl of about 
$0.05 \mathrm{~mm}$ in amplitude.

Of particular interest in this study is the use of the remaining coils of the AMB (when one fails) to provide a sideloading force to reduce rotor vibration. The test configuration consists of allowing coil 2 to fail and investigating the effects of sideloading on the system. Coils 1 and 3 are kept in the same electronic configuration as if no failure had occurred. However, the voltage in coil 4 is set to a value that will pull the shaft horizontally over to the auxiliary bearing. Two basic scenarios for rotor control during failure are considered. Figures 5.a - 5.c illustrate the rotor behavior under the conditions for the first scenario, when the rotor speed is maintained at a constant value. For each case, the AMB fails at $t_{f}=0.5$ seconds. There is some perturbation of the vertical motion of the rotor when the horizontal coil fails due to coupling of the horizontal and vertical motion (primarily) through the gyroscopic terms. However, the steady state vertical responses are not significantly affected. Figures 6.a - 6.c show the rotor behavior for the same failure conditions (with sideloading) as above for the second scenario, when the rotor is decelerated at a constant rate upon AMB failure. Again the response amplitudes tend to be lowest for smaller clearance values. It is observed that the responses may be quite high as the rotor contacts the auxiliary bearing, even with sideloading from the remaining coil. However, sideloading is shown to be quite effective in encouraging rotor contact that will dissipate the vibrational energy. The response amplitudes tend to decrease as the auxiliary bearing clearance is decreased. However from a practical perspective, there are definite design tradeoffs that must be considered. If the clearance is too small, the rotor may strike the auxiliary bearing during normal operation of the magnetic bearing if the rotor is perturbed. This tends to unnecessarily shorten the life of the auxiliary bearing. If the clearance is too large, the rotor vibration (upon AMB failure) will be excessively high and may damage the rotor or the magnetic bearing structure.

The effect the sideloading voltage on the system dynamics is particularly interesting. The magnetic bearing forces are nonlinear functions of the dynamic clearance and the current. If one coil fails and the remaining coils are used to provide sideloading, the natural approach might seem to issue a constant command voltage, with the objective of having a constant sideloading force at steady state. However, the inherently unstable behavior of the magnetic bearing without active control coupled with the vibrational

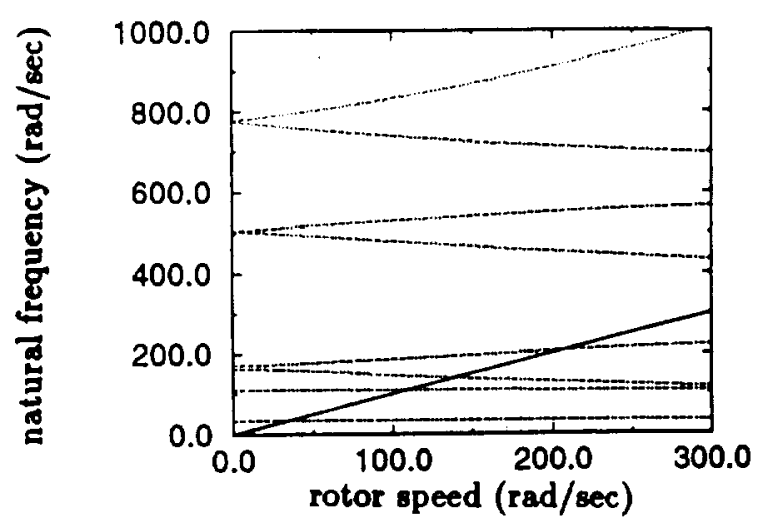

Figure 3 Rotor Natural Frequencies as a Function of Rotor Speed (for the parameters of Table 1)

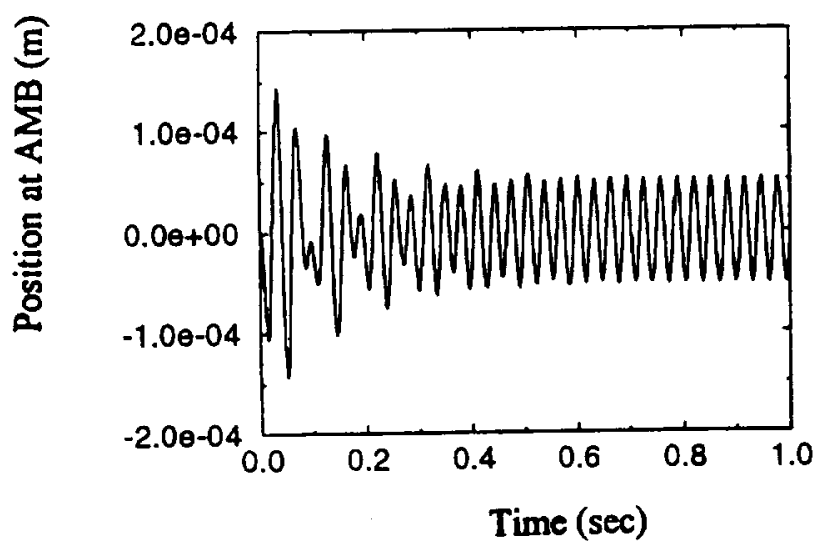

Figure 4 Rotor Response Without AMB Failure $\Omega=200 \mathrm{rad} / \mathrm{sec}, C_{L}=\frac{D}{2}$

effects between the auxiliary bearing and the rotor produces a severely unstable response. A remedy is to command a voltage that is proportional to the horizontal shaft displacement. That is, $v^{a}=v_{o}^{a}\left(D+x_{N_{B 2}}\right)$, where $v_{o}^{a}$ is a constant. With an ideal amplifier, any value of $v_{o}^{a}$ can be used with such a system. However due to the delay in the dynamics from the time constant of the power amplifier, unstable behavior may also result if $v_{0}^{a}$ is too large. Figure 7 shows the maximum $v_{o}^{a}$ that can be commanded with stable behavior as a function of power amplifier time constant. These results were developed by fitting a smooth curve through data points obtained by observing the behavior of the simulation model for various sideloading voltages and 


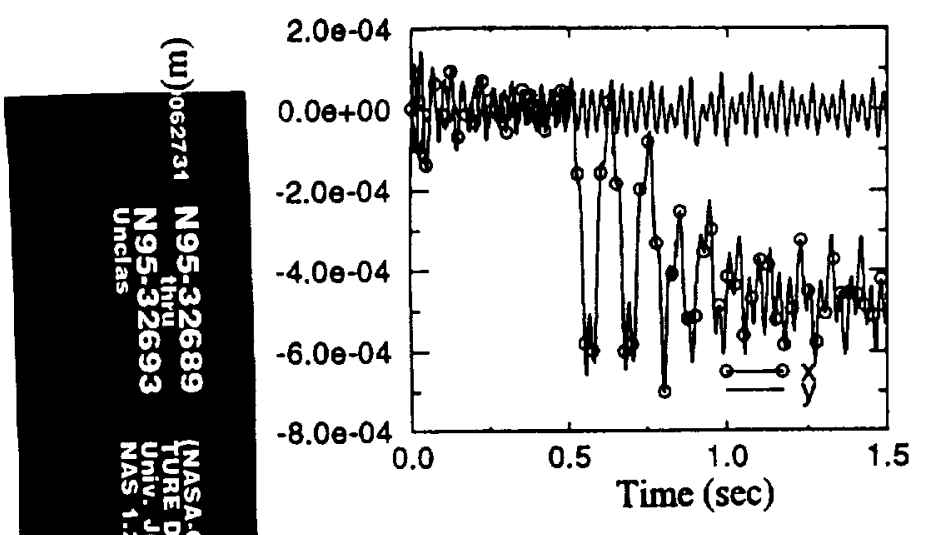

5.a Rotor Response With AMB Failure $\Omega=200 \mathrm{rad} / \mathrm{sec}, C_{L}=\frac{2 D}{3}$
$t_{f}=0.5, \alpha=0$

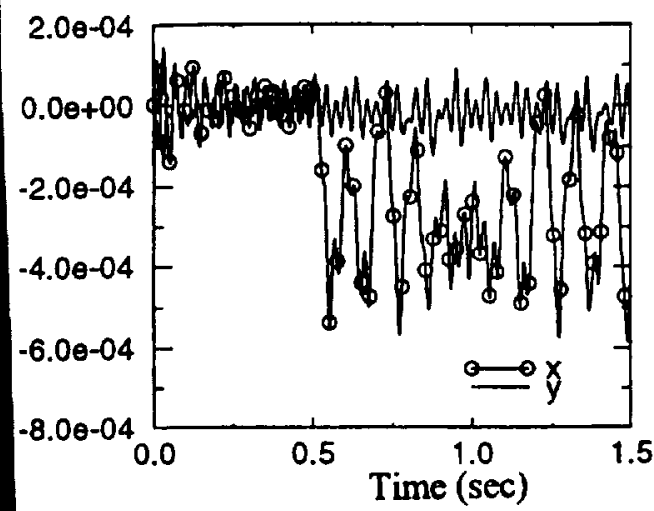

.b Rotor Response With AMB Failure $\begin{aligned} \Omega & =200 \mathrm{rad} / \mathrm{sec}, C_{L}=\frac{D}{2} \\ t_{f} & =0.5, \alpha=0\end{aligned}$

\& 8

time constants. It must be concluded er amplifier dynamics are a critical facselection of appropriate levels of bearing sideloading.

\section{CONCLUSION}

A simulation model has been developed for a magnetic bearing supported flexible rotor system with auxiliary bearings including frictional effects. The model has been described in detail with experimentally obtained model parameters. The response characteristics for a variety of system configurations were presented and discussed, including the effects of sideloading. Some guidelines were given for the selection of appropriate levels of sideloading.

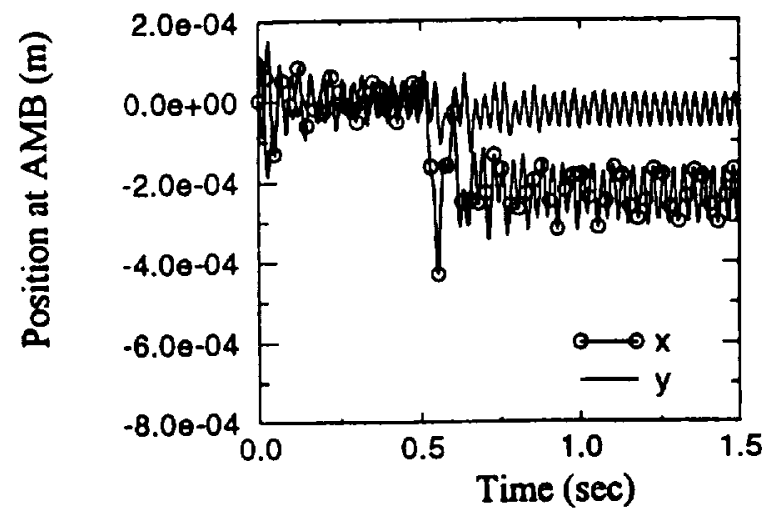

Figure 5.c Rotor Response With AMB Failure

$$
\begin{aligned}
\Omega & =200 \mathrm{rad} / \mathrm{sec}, C_{L}=\frac{D}{3} \\
t_{f} & =0.5, \alpha=0
\end{aligned}
$$

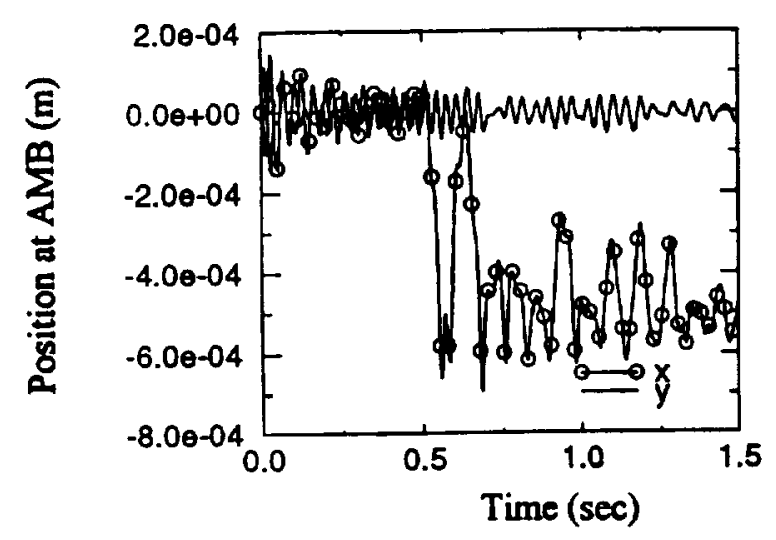

Figure 6.a Rotor Response With AMB Failure $\Omega=200 \mathrm{rad} / \mathrm{sec}, C_{L}=\frac{2 D}{3}$ $t_{f}=0.5, \alpha=-100$

\section{ACKNOWLEDGEMENT}

This work was supported by the National Aeronautics and Space Administration under Grant No. NAG3-1507. The Government has certain rights in this material. Special appreciation is expressed to Dr. Albert F. Kascak of NASA/Lewis Research Center.

\section{REFERENCES}

Gondhalekar, V., and Holmes, R., "Design of a Radial Electromagnetic Bearing for the Vibration Control of a Supercritical Shaft," Proceedings of the Institution of Mechanical Engineers, Vol. 198C, No. 16, pp. 235-242.

Habermann, H., and Liard, G., "An Active Magnetic Bearing System," Tribology Inter- 


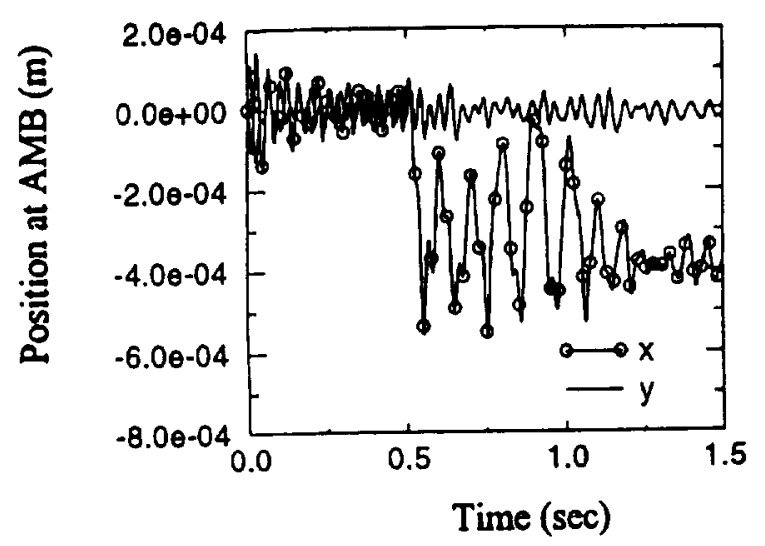

Figure 6.b Rotor Response With AMB Failure

$$
\begin{aligned}
\Omega & =200 \mathrm{rad} / \mathrm{sec}, C_{L}=\frac{D}{2} \\
t_{f} & =0.5, \alpha=-100
\end{aligned}
$$

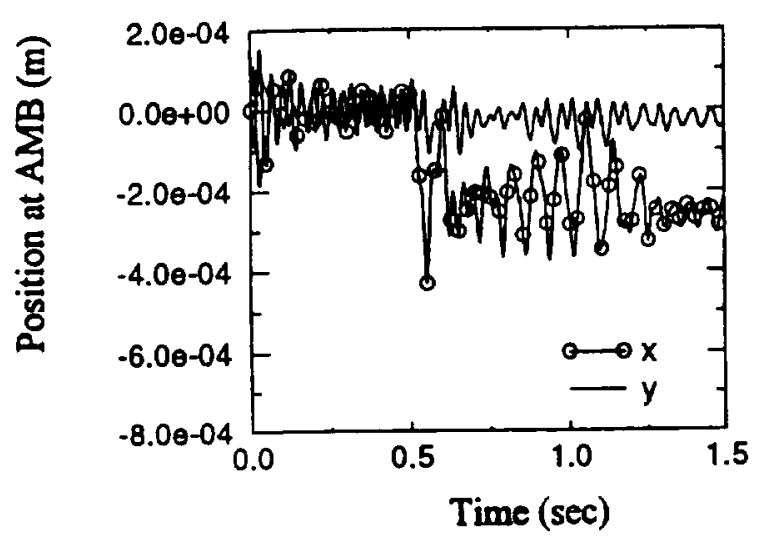

Figure 6.c Rotor Response With AMB Failure

$$
\begin{gathered}
\Omega=200 \mathrm{rad} / \mathrm{sec}, C_{L}=\frac{D}{3} \\
t_{f}=0.5, \alpha=-100
\end{gathered}
$$

national, April, 1980, pp. 85-89.

Humphris, R.R., Kelm, R.D., Lewis, D.W., and Allaire, P.E., "Effect of Control Algorithms on Magnetic Journal Properties," ASME Journal of Engineering for Gas Turbines and Power, Vol. 108, October, 1986, pp. 624-632.

Ishii, T., Kirk, R.G., "Transient Response Technique Applied To Active Magnetic Bearing Machinery During Rotor Drop," Rotating Machinery and Vehicle Dynamics, Vol. 35, 1991, pp. 191-199.

Lewis, David W., "Electro and Permanent Magnet Materials," Introduction To Magnetic Bearings: A Short Course, July 27-28, 1993.

Shafai, B., Beale, S., LaRocca, and Cusson,

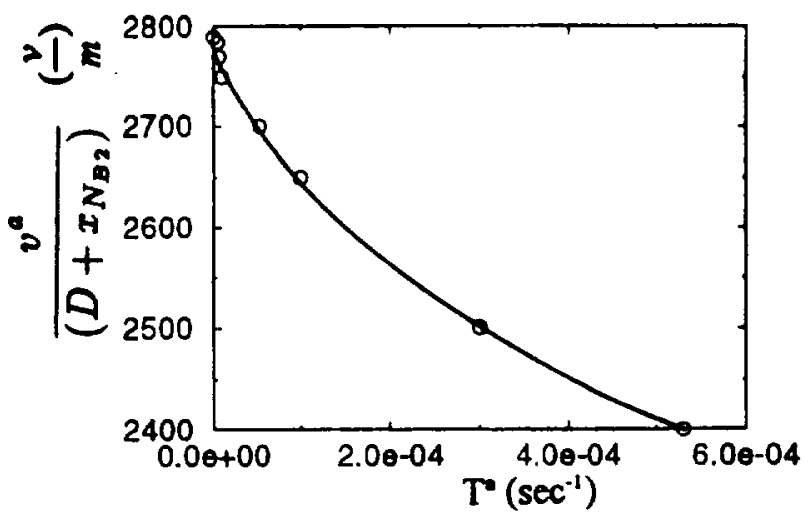

Figure 7 Maximum Sideloading Force as a Function of Power Amplifier Time Constant, $\tau^{a}$ (for parameters of Table 1)

E., "Magnetic Bearing Control Systems and Adaptive Forced Balancing," IEEE Control Systems, Vol. 14, No. 2, pp. 4-13.

\section{APPENDIX LINEAR CURRENT AND POSITION STIFFNESSES}

For design purposes, a linearized model for the magnetic bearing forces is needed.

The current stiffnesses are:

$$
\begin{aligned}
& K_{1}^{i}=\frac{\mu_{o} A k_{1}^{a} v_{1}^{b i} N_{T}^{2}}{2 D^{2}} \\
& K_{2}^{i}=\frac{\mu_{0} A k_{2}^{a} v_{2}^{b i} N_{T}^{2}}{2 D^{2}} \\
& K_{3}^{i}=\frac{\mu_{0} A k_{1}^{a} v_{1}^{b i} N_{T}^{2}}{2 D^{2}} \\
& K_{4}^{i}=\frac{\mu_{0} A k_{2}^{a} v_{2}^{b i} N_{T}^{2}}{2 D^{2}}
\end{aligned}
$$

The position stiffnesses are:

$$
\begin{aligned}
& K_{1}^{P}=\frac{\mu_{o} A\left(k_{1}^{a} v_{1}^{b i}\right)^{2} N_{T}^{2}}{2 D^{3}} \\
& K_{2}^{P}=\frac{\mu_{o} A\left(k_{2}^{a} v_{2}^{b i}\right)^{2} N_{T}^{2}}{2 D^{3}}
\end{aligned}
$$

